\title{
The nonperturbative contribution to asymptotic masses
}

\author{
Guy D. Moore $\oplus^{1, *}$ and Niels Schlusser $\circledast^{1,2, \dagger}$ \\ ${ }^{1}$ Institut für Kernphysik, Technische Universität Darmstadt, Schlossgartenstraße 2, \\ D-64289 Darmstadt, Germany \\ ${ }^{2}$ Department of Physics and Helsinki Institute of Physics, P.O. Box 64, FI-00014, \\ University of Helsinki, Helsinki, Finland
}

(Received 8 October 2020; accepted 19 October 2020; published 17 November 2020)

\begin{abstract}
In gauge theories, charged particles obey modified dispersion due to medium interactions (forward scattering), leading at high energies $E \geq T$ to an asymptotic mass-squared $m_{\infty}^{2}$. We calculate the infrared part of this mass nonperturbatively for the theory of the strong interactions, QCD, through a lattice treatment of its low-energy effective description, electrostatic QCD. Incorporation of these results into a nonperturbative determination of the effective thermal mass will require a still-incomplete next-to-leading order perturbative matching of this quantity to full QCD.
\end{abstract}

DOI: $10.1103 /$ PhysRevD.102.094512

\section{INTRODUCTION}

The quark-gluon-plasma (QGP) is an exotic state of matter created at collider facilities [1-4] and delivering important insights into the nature of strongly interacting matter. The experimental effort to measure the features of the QGP with the highest possible precision goes hand in hand with the theoretical goal of giving precise predictions for these very measurements. The properties of the QGP can in essence be divided into two classes: thermodynamics and transport properties. Typical examples for the former are the pressure, perturbatively determined up to $\mathcal{O}\left(g^{6} \ln g\right)$ [5-10] and investigated nonperturbatively on the lattice $[11,12]$, and charge susceptibilities and other quark-number correlations, which are well investigated on the lattice [13]. Since thermal equilibrium is time-translation invariant, these results can be derived in Euclidean time, which simplifies the calculations considerably. In contrast, the calculation of transport properties usually requires an explicit treatment of Minkowski time, which is conceptually more demanding. Nevertheless, there has been significant progress in recent years, e.g., for the shear viscosity $\frac{\eta}{s}$ $[14,15]$ or the thermal photon production rate $[16,17]$.

Another interesting transport phenomenon to investigate is how the medium modifies a jet, whose theoretical treatment is, e.g., summarized in [18]. For our purpose,

\footnotetext{
guy.moore@physik.tu-darmstadt.de

nschlusser@theorie.ikp.physik.tu-darmstadt.de
}

Published by the American Physical Society under the terms of the Creative Commons Attribution 4.0 International license. Further distribution of this work must maintain attribution to the author(s) and the published article's title, journal citation, and DOI. Funded by SCOAP. jets are strongly interacting particles that carry a large momentum and move at (almost) the speed of light. Because of the large momentum scale, asymptotic freedom of QCD suggests that the strong coupling constant $g$ is small $[19,20]$, and that they can be well described by an expansion in powers of $g$. In traversing through the medium, however, they receive highly nontrivial modifications [21,22], pictured by a large number of soft bumps between jet and medium constituents. This interaction eventually leads to jet broadening, i.e., a broadening of the jet's transversal momentum distribution. Related to that, jet quenching refers to a jet losing energy to the medium $[23,24]$, predominantly via stimulated emission due to multiple soft scatterings with medium constituents.

A quantity that influences both phenomena is the asymptotic mass. It was already found by Klimov and by Weldon that massless particles, be it gluons $[25,26]$ or fermions $[27,28]$, tend to follow the dispersion relation of massive particles

$$
\omega_{\boldsymbol{p}}^{2}=\boldsymbol{p}^{2}+m_{\boldsymbol{p}}^{2},
$$

with their mass $m_{p}^{2}$ depending on the momentum $\boldsymbol{p}$ and generated by the interaction of the particle with the medium (essentially, forward scattering). At large momenta, the particle's velocity in the plasma frame approaches the speed of light $v \rightarrow(1, \hat{\boldsymbol{p}})$, and this thermal mass approaches a constant which we will call the asymptotic mass $m_{\infty}^{2}$. At the leading perturbative order, $m_{\infty}^{2}$ can be computed from the imaginary part of the transverse hard-thermal-loopresummed self-energy $\Pi_{\mathrm{T}}$ near the light cone $[29,30]$. The gluon's asymptotic mass, e.g., reads [31]

$$
m_{\infty}^{2} \equiv \Pi_{\mathrm{T}}\left(k_{0}=k\right)=\frac{m_{\mathrm{D}}^{2}}{2}
$$


to first perturbative approximation. Here $m_{\mathrm{D}}^{2}=\left(2 N_{c}+\right.$ $\left.N_{\mathrm{f}}\right) g^{2} T^{2} / 6$ is the Debye screening mass squared.

The expression (1.2) can be generalized to a particle in an arbitrary representation $\mathrm{R}$ and related to a combination of two condensates [32,33], a gauge contribution $Z_{\mathrm{g}}$, and a fermionic contribution $Z_{\mathrm{f}}$,

$$
m_{\infty}^{2}=g^{2} C_{\mathrm{R}}\left(Z_{\mathrm{g}}+Z_{\mathrm{f}}\right),
$$

with the (running) gauge coupling $g$ and the Casimir operator of the representation $C_{\mathrm{R}}$ of the particle that makes up the jet. The two condensates are $[32,33]$

$$
\begin{aligned}
& Z_{\mathrm{f}} \equiv \frac{1}{2 d_{\mathrm{R}}}\left\langle\bar{\psi} \frac{v_{\mu} \gamma^{\mu}}{v \cdot D} \psi\right\rangle \\
& Z_{\mathrm{g}} \equiv-\frac{1}{g^{2} d_{\mathrm{A}}}\left\langle v_{\sigma} F^{\sigma \mu} \frac{1}{(v \cdot D)^{2}} v_{\rho} F^{\rho}{ }_{\mu}\right\rangle,
\end{aligned}
$$

where $d_{\mathrm{A}}=N_{c}^{2}-1$ is the dimension of the adjoint representation, in which the gauge bosons live, and $d_{\mathrm{R}}$ is the dimension of the representation $\mathrm{R}$ of the fermion, e.g., the fundamental representation of $S U(3)$ for quarks in QCD, and the angular brackets represent an average over all

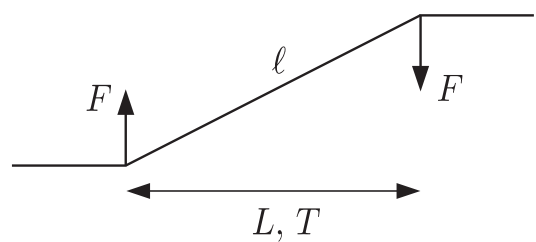

FIG. 1. Pictorial motivation for the form of the correlator (1.5).

thermal fluctuations. Note that our definition of the QCD field strength $F^{\sigma \mu}$ is the geometrical one, $F^{\mu \nu}=i\left[D^{\mu}, D^{\nu}\right]$ with $D^{\mu}=\partial^{\mu}-i A^{\mu} ; g^{2}$ is absorbed into the coefficient on the field strength in the action, $S=\int \frac{1}{2 g^{2}} \operatorname{Tr} F^{2}+\ldots$. These condensates represent the contribution of forward scattering in the medium, with $1 /(v \cdot D)$ or $(v \cdot D)^{2}$ accounting for the lightlike (eikonalized) propagation through the medium and $\bar{\psi}, \psi$ or $v_{\sigma} F^{\sigma \mu}$ representing an interaction with a medium excitation. The leading-order, long-distance approximation to these condensates reproduces the forward-scattering part of the hard-thermal-loop effective action along the light cone.

The gauge part of (1.3) can be related to an integral over a correlator in position space [15]

$$
Z_{\mathrm{g}}=-\frac{1}{g^{2} d_{\mathrm{A}}} \int_{0}^{\infty} \mathrm{d} x_{+} x_{+}\left\langle v_{k \mu} F_{a}^{\mu \nu}\left(x_{+}, 0,0_{\perp}\right) U_{\mathrm{A}}^{a b}\left(x_{+}, 0,0_{\perp} ; 0,0,0_{\perp}\right) v_{k \rho} F_{b \nu}^{\rho}\left(0,0,0_{\perp}\right)\right\rangle,
$$

where the $x_{+}$integration and the modified adjoint Wilson line $U_{\mathrm{A}}^{a b}$ arise from $1 /(v \cdot D)^{2}$. Locations in space-time are described by three-vectors $\left(x_{+}, x_{-}, x_{\perp}\right)$ in light-cone coordinates. Because the jet is highly relativistic, we have $x_{-}=$ $t-z=0$ in the plasma frame. The problem therefore effectively reduces to three dimensions, two in the transverse plane $x_{\perp}$, where rotational invariance is present, and a coordinate that encompasses the elapsed time $t$ for the jet as well as its covered distance $z$ in the medium $x_{+}=(t+z) / 2 \equiv L$. We use the convention that the $\mathrm{z}$-component of $\boldsymbol{k}$ is the jet's direction of propagation, so $v=(1,0,0,1)$.

At this point, we would like to briefly build up some intuition for the specific form of (1.5). We can distinguish two sorts of jet-medium interactions. First, there is true scattering, where the transverse momentum of the jet particle changes; this is described by the transverse momentum broadening kernel $C\left(\boldsymbol{b}_{\perp}\right)$ [22]. But there is also forward scattering, where the jet particle temporarily changes direction before scattering back into its original state (or more technically, a scattering creates an amplitude in a different momentum state, which subsequently scatters again to return to the original momentum, introducing a phase shift). Such forward scattering is what causes a dispersion correction. It can be understood qualitatively as the jet particle making a scattering-induced detour, as pictured in Fig. 1. The sum of all such forward-scattering possibilities induces an effective mass term [23].

Relativistic kinematics tells us the length of the detour,

$$
\ell^{2}=L^{2}+\frac{F^{2} T^{4}}{m^{\prime 2} \gamma(v)} \equiv L^{2}+\Delta \ell^{2},
$$

where $m^{\prime}$ is a dummy jet mass that will eventually drop out of the calculation, $\gamma(v)$ is the gamma factor of the jet at velocity $v$, and $T$ is the time it takes for the jet to cover the distance $L$. If we consider this problem from a frame of reference in which we do not take into account the additional detour, the kinematic invariant reads ${ }^{1}$

$$
\begin{gathered}
p^{\mu} p_{\mu}=m^{\prime 2} \gamma^{2}(v)\left(-1+\frac{\ell^{2}-\Delta \ell^{2}}{T^{2}}\right) \\
\stackrel{T \rightarrow \ell, m^{\prime} \rightarrow 0}{\longrightarrow}-F^{2} \ell^{2} \approx-F^{2} L^{2}
\end{gathered}
$$

in the ultrarelativistic limit, in which also the contribution of longitudinal forces to the effective mass vanishes. Consequently, the (transversal) gauge force must appear twice in the correlator resulting in the mass. Furthermore, a full quantum treatment requires a sum over all possible

\footnotetext{
${ }^{1}$ We use the Minkowski metric with the negative sign in the time component.
} 
paths instead of just considering one as in Fig. 1. Hence, our estimate for the effective mass yields

$$
\sum_{L} F^{2} L^{2} \rightarrow \int \mathrm{d} L L F^{2} \sim m_{\infty}^{2},
$$

reflecting the qualitative features of (1.5).

The state-of-the-art perturbative result for $Z_{\mathrm{g}}$ at next-toleading-order (NLO) [33] is

$$
Z_{\mathrm{g}}=Z_{\mathrm{g}}^{\mathrm{LO}}+\delta Z_{\mathrm{g}}=\frac{T^{2}}{6}-\frac{T m_{\mathrm{D}}}{2 \pi} .
$$

The Debye mass [26], in turn, depends on the coupling $g$ : in QCD $\left(N_{c}=3\right)$; it reads

$$
m_{\mathrm{D}}^{2}=g^{2} T^{2}\left(1+\frac{N_{\mathrm{f}}}{6}\right)+\mathcal{O}\left(g^{4}\right) .
$$

Formally, the NLO correction is therefore suppressed by a single power of the coupling $g$. Numerically, it is not suppressed even at $T=100 \mathrm{GeV}$ temperatures, indicating a very poor convergence for the perturbative series. This is a familiar situation for perturbative calculations in hot QCD, and as usual, it arises because there are large corrections arising from the large-occupancy, infrared (IR) sector of QCD. Specifically, the convergence of the perturbative series is spoiled by the high occupancy of the gluon 0-Matsubara-frequency mode. When this mode circulates in a loop, it introduces an additional factor of $n_{\mathrm{B}} \sim 1 / g$ [34-36]. A possible solution to this problem is provided by treating the badly convergent contributions separately in a framework called electrostatic quantum chromodynamics (EQCD) [37].

In contrast, the situation for $Z_{\mathrm{f}}$ appears to be better under control. Fermions do not feature a 0 -mode so the above reasoning does not apply here. Indeed, it was found that [33]

$$
Z_{\mathrm{f}}=Z_{\mathrm{f}}^{\mathrm{LO}}+\delta Z_{\mathrm{f}}=\frac{T^{2}}{12}+0,
$$

so the assumption of a convergent series for the fermionic part of $m_{\infty}^{2}$ may be justified.

According to [38], correlators like (1.4) can be computed in a setup that quantizes the plasma on the light cone. Their dynamics is dominated by multiple soft scatterings that have to be resummed. But because they are infrared, they can be computed in three-dimensional EQCD instead of four-dimensional full QCD in real time, with modified Wilson lines that account for the parallel transport of the gauge configurations. In the past, this technique has been predominantly used for the computation of the transverse collision kernel $C\left(\boldsymbol{b}_{\perp}\right)$ [38-40]. Similarly to the calculation of $C\left(\boldsymbol{b}_{\perp}\right)$, the EQCD operator agrees with its full QCD equivalent only in the IR regime where EQCD is supposed to be a valid description of full QCD. In the ultraviolet regime, however, a difference between the two operators will emerge. This difference should be under perturbative control and can be included as part of a matching calculation between thermal QCD and EQCD, when the matching is extended to include the operators of Eq. (1.5).

In the course of the present work, we will evaluate the soft operator in EQCD and leave the purely perturbative matching calculation for later investigation. As for $C\left(\boldsymbol{b}_{\perp}\right)$, perturbation theory, even in EQCD, is not always sufficient to deal with the nonperturbative nature of some hightemperature phenomena. Sometimes, it is necessary to solve EQCD directly on the lattice, which has been done recently for $C\left(\boldsymbol{b}_{\perp}\right)[39,40]$. This will also be our method of choice for the determination of the soft part of asymptotic jet masses in the following.

We address the problem as follows. In Sec. II, we outline how we would compute $Z_{\mathrm{g}}$ if there were no interactions. To this end, we briefly introduce how our setup translates into EQCD, show how the observables appear in this theory, and finally present the free solutions. Solving the full interacting theory requires discretizing the theory and the corresponding observables on the lattice, done in Sec. III. This part of the present work also outlines how to deal with the contributions of Eq. (1.5) at the shortest and longest distances, where the lattice determination becomes problematic. Section IV involves a detailed presentation of our results together with a thorough discussion and, where possible, quantification of the errors that our results are fraught with. The consequences of our results and how to improve on them are discussed in Sec. V. The appendix provides a detailed overview of our lattice configurations.

\section{FORMULATION OF THE PROBLEM}

\section{A. Electrostatic QCD}

EQCD is an effective field theory for hot QCD. At high temperatures, the scaling behavior changes, for lengths longer than $1 / T$ or energies lower than $T$, to the behavior of a 3D theory, which is more strongly coupled in the IR. Within the Matsubara formalism for describing thermal $\mathrm{QCD}$, this arises because the usual frequency integral $\int d \omega / 2 \pi$ is replaced by a discrete sum $T \sum_{\omega=2 n \pi T}$, with the $n=0$ (zero Matsubara frequency) mode massless (at tree level), leading to an $\mathcal{O}(2 \pi T / p)$ enhancement of small momentum $p$ parts of diagrams. For the electric sector, this is cut off by the Debye mass, but for the magnetic sector it is not cut off until magnetic degrees of freedom become strongly coupled and confine. For an overview, see Refs [34-36]. The idea of EQCD is to integrate out-in a Wilsonian renormalization group sense-all Matsubara modes in QCD except for these 0-modes, which can then be treated nonperturbatively. Since there is only one Matsubara mode left, one has effectively eliminated the Euclidean time direction. Therefore, the transition to EQCD is often called dimensional reduction. A time direction that only consists of one time layer does not allow for derivatives in the time direction any more. 
TABLE I. EQCD parameters at four different temperatures and number of fermion flavors.

\begin{tabular}{lcll}
\hline \hline$T$ & $n_{\mathrm{f}}$ & \multicolumn{1}{c}{$x$} & \multicolumn{1}{c}{$y$} \\
\hline $250 \mathrm{MeV}$ & 3 & 0.08896 & 0.452423 \\
$500 \mathrm{MeV}$ & 3 & 0.0677528 & 0.586204 \\
$1 \mathrm{GeV}$ & 4 & 0.0463597 & 0.823449 \\
$100 \mathrm{GeV}$ & 5 & 0.0178626 & 1.64668 \\
\hline \hline
\end{tabular}

Consequently, this direction no longer features gauge symmetry, which is what allows the temporal gauge field $A^{0}$, now responsible for all electrical phenomena, to acquire a mass $m_{\mathrm{D}}^{2}$. Loop level effects induce a self-coupling for this field, denoted as $\lambda$. A scale is set by the now dimensionful gauge coupling $g_{3 \mathrm{~d}}^{2}=g^{2} T$. The full action of EQCD in the continuum reads

$$
\begin{aligned}
S_{\mathrm{EQCD}, \mathrm{c}}= & \int \mathrm{d}^{3} x\left(\frac{1}{2 g_{3 \mathrm{~d}}^{2}} \operatorname{Tr} F^{i j} F^{i j}+\operatorname{Tr} D^{i} \Phi D^{i} \Phi\right. \\
& \left.+m_{\mathrm{D}}^{2} \operatorname{Tr} \Phi^{2}+\lambda\left(\operatorname{Tr} \Phi^{2}\right)^{2}\right) .
\end{aligned}
$$

The parameters in (2.1) can be related to the full theory via a perturbative matching procedure [37]. We employ a $\mathcal{O}\left(g^{4}\right)$-accurate result [41]. Input parameters are the temperature $T$ and the number of dynamical massless fermion flavors $N_{\mathrm{f}}$ translating into the pair of (dimensionless) EQCD parameters $x \equiv \lambda / g_{3 \mathrm{~d}}^{2}$ and $y \equiv m_{\mathrm{D}}^{2} /\left.g_{3 \mathrm{~d}}^{4}\right|_{\mu=g_{3 \mathrm{~d}}^{2}}$. Note that $m_{\mathrm{D}}^{2}$ varies logarithmically with scale due to two-loop effects; therefore, in defining $y$, we must specify the renormalization point. Following convention, we choose $\mu=g_{3 \mathrm{~d}}^{2}$. We will consider the values given in Table I. EQCD also has a nontrivial phase structure $[42,43]$, with a transition between a $\mathbb{Z}_{3}$-symmetric and broken phase that is of second order at large $x$ and turns into a first order one for smaller $x$, including all physically relevant $x$ values. Note the $(x, y)$-pairs which describe full QCD prove to lie in the region where the $3 \mathrm{D}$ theory prefers the $\mathbb{Z}_{3}$-broken phase. Therefore, we are to consider metastable $\mathbb{Z}_{3}$-symmetric states in a parameter range where the broken phase is actually thermodynamically preferred. Thus, choosing sufficiently large volumes is crucial; they keep the system from accidentally falling into the broken phase. It is believed that dimensional reduction to EQCD is valid for all $T \gtrsim 2 T_{\mathrm{c}}$ and possibly even further down [44], where $T_{\mathrm{c}}$ is the transition temperature of full QCD.

\section{B. Observables}

As already mentioned, one promotes (1.5) to EQCD by replacing Wilson lines along the light cone with their modified EQCD counterparts [38],

$$
\begin{aligned}
& \tilde{U}_{\mathrm{R}}\left(x_{\perp}, L ; x_{\perp}, 0\right) \\
& =\mathrm{P} \exp \left(\int_{0}^{L} \mathrm{~d} z\left(i A_{z}^{a}\left(x_{\perp}, z\right)+g_{3 \mathrm{~d}} \Phi^{a}\left(x_{\perp}, z\right)\right) T_{\mathrm{R}}^{a}\right)
\end{aligned}
$$

and $F^{i 0}$ with $D^{i} \Phi$. Applying rotational invariance in the transversal plane, we find

$$
\begin{aligned}
Z_{\mathrm{g}, 3 \mathrm{~d}}= & -\frac{2}{d_{\mathrm{A}}} \int_{0}^{\infty} \mathrm{d} L L\left(\left\langle\left(D^{x} \Phi(L)\right)_{a} \tilde{U}_{\mathrm{A}}^{a b}(L, 0)\left(D^{x} \Phi(0)\right)_{b}\right\rangle\right. \\
& \left.+\left\langle F_{a}^{x z}(L) \tilde{U}_{\mathrm{A}}^{a b}(L, 0) F_{b}^{x z}\right\rangle\right) .
\end{aligned}
$$

This correlation function is UV safe within EQCD; here it is essential that the modified Wilson loop of Eq. (2.2) is used, and not the standard Wilson loop without the $\Phi^{a} T^{a}$ term in Eq. (2.2); the standard Wilson loop contains a logarithmically UV divergent perimeter law suppression which is canceled by the inclusion of the scalar.

As a matter of choice, we work in the fundamental representation of the correlators, related to the adjoint ones via

$$
\begin{aligned}
& \left\langle\left(D^{x} \Phi(L)\right)_{a} \tilde{U}_{\mathrm{A}}^{a b}(L ; 0)\left(D^{x} \Phi(0)\right)_{b}\right\rangle \\
& \quad=2 \operatorname{Tr}\left\langle D^{x} \Phi(L) \tilde{U}_{\mathrm{F}}(L ; 0) D^{x} \Phi(0) \tilde{U}_{\mathrm{F}}^{-1}(L ; 0)\right\rangle \\
& \left\langle F_{a}^{x z}(L) \tilde{U}_{\mathrm{A}}^{a b}(L ; 0) F_{b}^{x z}\right\rangle \\
& \quad=2 \operatorname{Tr}\left\langle F^{x z}(L) \tilde{U}_{\mathrm{F}}(L ; 0) F^{x z}(0) \tilde{U}_{\mathrm{F}}^{-1}(L ; 0)\right\rangle .
\end{aligned}
$$

In the following, we will drop the tilde on top of the modified Wilson lines $\tilde{U}$ for the sake of readability and write half the right-hand side of (2.4) as $\operatorname{Tr}\left\langle E(L) U E(0) U^{-1}\right\rangle$ and half the right-hand side of (2.5) as $\operatorname{Tr}\left\langle B(L) U B(0) U^{-1}\right\rangle$.

Let us stress again that the argument of Eq. (2.3) agrees with that of Eq. (1.5) only up to IR-safe terms which can and must be determined in a matching calculation between full thermal QCD and EQCD. This calculation has not been carried out for these specific operators, though it should proceed along similar lines to the matching already conducted for $C\left(\boldsymbol{b}_{\perp}\right)$ in $[15,38]$.

Through insertions of the scale $g_{3 \mathrm{~d}}^{2}$, everything can be recombined into dimensionless quantities and we end up with the master formula for our observable

$$
\begin{aligned}
\frac{Z_{\mathrm{g}}}{g_{3 \mathrm{~d}}^{4}}= & -\frac{1}{2} \int_{g_{3 \mathrm{~d}}^{2} L_{\min }^{\mathrm{E}}}^{\infty} \mathrm{d}\left(g_{3 \mathrm{~d}}^{2} L\right) g_{3 \mathrm{~d}}^{2} L \frac{\operatorname{Tr}\left\langle D^{x} \Phi\left(g_{3 \mathrm{~d}}^{2} L\right) U_{\mathrm{F}}\left(g_{3 \mathrm{~d}}^{2} L ; 0\right) D^{x} \Phi\left(g_{3 \mathrm{~d}}^{2} L\right) U_{\mathrm{F}}^{-1}\left(g_{3 \mathrm{~d}}^{2} L ; 0\right)\right\rangle}{g_{3 \mathrm{~d}}^{6}} \\
& -\frac{1}{2} \int_{g_{3 \mathrm{~d}}^{2} L_{\min }^{\mathrm{B}}}^{\infty} \mathrm{d}\left(g_{3 \mathrm{~d}}^{2} L\right) g_{3 \mathrm{~d}}^{2} L \frac{\operatorname{Tr}\left\langle F^{x z}\left(g_{3 \mathrm{~d}}^{2} L\right) U_{\mathrm{F}}\left(g_{3 \mathrm{~d}}^{2} L ; 0\right) F^{x z}(0) U_{\mathrm{F}}^{-1}\left(g_{3 \mathrm{~d}}^{2} L ; 0\right)\right\rangle}{g_{3 \mathrm{~d}}^{8}},
\end{aligned}
$$


where $g_{3 \mathrm{~d}}^{2} L_{\min }^{\mathrm{E}}$ and $g_{3 \mathrm{~d}}^{2} L_{\min }^{\mathrm{B}}$ are two minimal separations at which we can measure our observable in the EQCD effective picture on the lattice. Beyond that, we have to rely on analytical solutions, as we will elaborate in Sec. III.

\section{Free solutions}

The short-distance behavior of the full, continuumextrapolated correlators in (2.4) and (2.5) is expected to match the perturbative expectations and eventually, at sufficiently small separations, the free solutions. Since they not only provide an important cross-check for our data, but also will be of practical use later, we will briefly present their instructive but simple derivation in the following.

We assume the transverse direction to be oriented along the $x$-axis for simplicity. At tree-level, i.e., at $\mathcal{O}\left(g_{3 \mathrm{~d}}^{0}\right),(2.4)$ reduces to

$$
\begin{aligned}
& \operatorname{Tr}\left\langle D^{x} \Phi(L) U_{\mathrm{F}}(L, 0) D^{x} \Phi(L) U_{\mathrm{F}}^{-1}(L, 0)\right\rangle \\
& =\left.\partial_{x}^{2} \operatorname{Tr}\langle\Phi(x, L) \Phi(0,0)\rangle\right|_{x \rightarrow 0} \\
& =\left.C_{\mathrm{A}} C_{\mathrm{F}} \partial_{x}^{2}\left(\frac{\mathrm{e}^{-m_{\mathrm{D}} \sqrt{x^{2}+L^{2}}}}{4 \pi \sqrt{x^{2}+L^{2}}}\right)\right|_{x \rightarrow 0} \\
& =-\frac{\mathrm{e}^{-m_{\mathrm{D}} L}}{\pi L^{3}}\left(1+m_{\mathrm{D}} L\right)
\end{aligned}
$$

specializing to $S U(3)$ in the last step, and in a similar fashion for (2.5),

$$
\begin{aligned}
\operatorname{Tr} & \left.F^{x z}(L) U_{\mathrm{F}}(L, 0) F_{x z} U_{\mathrm{F}}^{-1}(L, 0)\right\rangle \\
= & \left.\partial_{x}^{2} \operatorname{Tr}\left\langle A_{z}(x, L) A_{z}(0,0)\right\rangle\right|_{x \rightarrow 0} \\
& +\left.\partial_{z}^{2} \operatorname{Tr}\left\langle A_{x}(x, z) A_{x}(0,0)\right\rangle\right|_{x \rightarrow 0, z=L} \\
= & -\frac{C_{\mathrm{A}} C_{\mathrm{F}}}{4 \pi L^{3}}+\frac{2 C_{\mathrm{A}} C_{\mathrm{F}}}{4 \pi L^{3}}=\frac{1}{\pi L^{3}} .
\end{aligned}
$$

Plugging these results into (1.5), we see that the $\mathcal{O}\left(L^{-3}\right)$ and $\mathcal{O}\left(L^{-2}\right)$ parts cancel. The dominant short-distance behavior is of $\mathcal{O}(1 / L)$, which when integrated over $L \mathrm{~d} L$ leads to a finite short-distance behavior in $Z_{\mathrm{g}}$. The superrenormalizable nature of EQCD means that, at the next loop order, the small- $L$ behavior can be at worst $\mathcal{O}\left(L^{-2}\right)$. However, if there is no cancellation between the electric and magnetic contributions at this order, there could be complications in the short-distance behavior, leading potentially to logarithmic short-distance contributions in Eq. (2.3). If this is the case, their role must be clarified by the matching to the $4 \mathrm{D}$ theory.

\section{COMPUTATIONAL DETAILS}

\section{A. Lattice implementation}

Our lattice implementation agrees with the one in [40], based on a modification of Martin Lüscher's
openQCD-1.6 [45]. Due to [46,43], we are able to discretize the EQCD action on a three-dimensional spatial lattice without any errors at $\mathcal{O}(a)$. As an update algorithm, we used a composite of two heatbath sweeps followed by four overrelaxation sweeps through the volume in a checkerboard order. To improve the signal-to-noise ratio, we applied the multilevel algorithm $[47,48]$, dividing the volume into four subvolumes along the $z$-axis and updating each subvolume 80 times before a sweep through the entire volume took place.

\section{B. Observables on the lattice and their continuum extrapolation}

For the $F^{x z}$-insertions in (2.5), we use the standard clover discretization of the field-strength tensor [49,50], with four $1 \times 1$ clover leaves. The scalar field derivatives $D^{x} \Phi$ in (2.4) are discretized as

$$
\begin{aligned}
D^{x} \Phi(\boldsymbol{x})= & \frac{1}{2 a}\left(U(\boldsymbol{x}) \Phi\left(\boldsymbol{x}+a \boldsymbol{e}_{x}\right) U^{\dagger}(\boldsymbol{x})\right. \\
& \left.-U^{\dagger}\left(\boldsymbol{x}-a \boldsymbol{e}_{x}\right) \Phi\left(\boldsymbol{x}-a \boldsymbol{e}_{x}\right) U\left(\boldsymbol{x}-a \boldsymbol{e}_{x}\right)\right),
\end{aligned}
$$

keeping in mind that the rescaling of the $\Phi$-fields to their lattice equivalents yields another factor of $1 / a$. Note that the clover magnetic field operator is spatially larger than the electric field operator we use; therefore, its $\mathcal{O}\left(a^{2}\right)$ corrections are larger, and we need more lattice spacings of separation between the $B$ operators before continuum behavior sets in than for the $E$ operators. Consequently, we cannot pursue as short distances in the $\operatorname{Tr}\left\langle B(L) U B(0) U^{-1}\right\rangle$ correlators as in the $\operatorname{Tr}\left\langle E(L) U E(0) U^{-1}\right\rangle$ correlators.

All of our observables are tree-level $\mathcal{O}\left(a^{2}\right)$ accurate. Nevertheless, there are still $\mathcal{O}(a)$ discretization effects present generated by loop effects in lattice perturbation theory. These generate multiplicative $\mathcal{O}(a)$ corrections, which we will denote by $Z_{\mathrm{E}}$ and $Z_{\mathrm{B}}$ in the following. For details about the discretization of the modified Wilson line, we again refer to [40,39]. The $\mathcal{O}(a)$ renormalization of an isolated modified Wilson line, or two well-separated modified Wilson lines, was calculated analytically in [51]. We use the resulting prescription, which is to scale $A^{0}$ in Eq. (2.2) by a $Z$ factor which is determined there. However, for the current case, this is insufficient, since there are new UV effects which can occur because the two modified Wilson lines in Eqs. (2.4) and (2.5) are coincident. This will lead to new, so far uncalculated, $\mathcal{O}(a)$ perimeter-law renormalizations of the Wilson line, which must be accounted for. The overall lattice expressions eventually read

$$
\begin{aligned}
& \frac{\operatorname{Tr}\left\langle E(L) U E(0) U^{-1}\right\rangle_{\text {latt }}}{g_{3 \mathrm{~d}}^{6} a^{3}} \\
& =\left(\frac{\operatorname{Tr}\left\langle E(L) U E(0) U^{-1}\right\rangle_{\text {cont }}}{g_{3 \mathrm{~d}}^{6}}+\mathcal{O}\left(a^{2}\right)\right) \mathrm{e}^{g_{3 \mathrm{~d}}^{4} a L Z_{\mathrm{P}}}\left(1+g_{3 \mathrm{~d}}^{2} a Z_{\mathrm{E}}\right),
\end{aligned}
$$




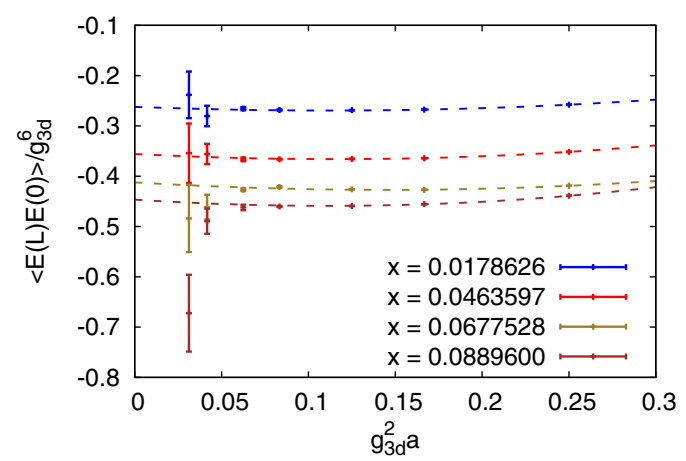

(a)

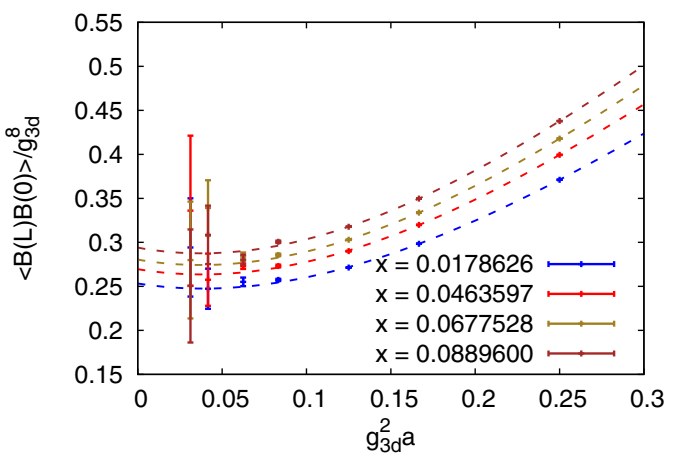

(b)

FIG. 2. Two continuum limits for $g_{3 \mathrm{~d}}^{2} L=1.0$ at all four considered temperatures. (a) $\frac{\operatorname{Tr}\left\langle E(L) U E(0) U^{-1}\right\rangle}{g_{3 \mathrm{~d}}^{6}}$ (b) $\frac{\operatorname{Tr}\left\langle B(L) U B(0) U^{-1}\right\rangle}{g_{3 \mathrm{~d}}^{8}}$.

$$
\begin{aligned}
& \frac{\operatorname{Tr}\left\langle B(L) U B(0) U^{-1}\right\rangle_{\text {latt }}}{g_{3 \mathrm{~d}}^{8} a^{4}} \\
& =\left(\frac{\operatorname{Tr}\left\langle B(L) U B(0) U^{-1}\right\rangle_{\text {cont }}}{g_{3 \mathrm{~d}}^{8}}+\mathcal{O}\left(a^{2}\right)\right) \\
& \quad \times \mathrm{e}^{g_{3 \mathrm{~d}}^{4} a L Z_{\mathrm{P}}}\left(1+g_{3 \mathrm{~d}}^{2} a Z_{\mathrm{B}}\right) .
\end{aligned}
$$

An analytical calculation of $Z_{\mathrm{E}}, Z_{\mathrm{B}}$, and $Z_{\mathrm{P}}$ is probably possible but appears to be technically somewhat complex, since the Wilson line operator is an extended object. However, by analyzing what diagrams could give rise to such linear in $a$ effects, we find that the rescalings $Z_{\mathrm{B}}$ and $Z_{\mathrm{E}}$ do not depend on the length $L$ (provided it is long in lattice units), and the perimeter contribution $Z_{\mathrm{P}}$ is strictly linear in $L$. Furthermore, the EQCD parameters $x$ and $y$ would first enter at a higher loop order than the NLO effect giving rise to $\mathcal{O}(a)$ corrections. Therefore, the $Z_{\mathrm{E}}, Z_{\mathrm{B}}$, and $Z_{\mathrm{P}}$ factors are common to all $(x, y)$ values and all separations. They represent only three total fitting parameters over all of our data at multiple $(x, y)$ and $L$ values. Such global parameters make the fit more complicated, introducing correlations between the fits to multiple lattice spacings, separations, and $(x, y)$ pairs. This made the fitting procedure somewhat unstable, which we cured by using a finite-difference solver to minimize $\chi^{2}$. The correlations between all data points must also be considered when performing the final numerical integrations. But because we only lose fitting 3 degrees of freedom over all data, the impact on the overall error budget is relatively modest.

Figure 2 shows a generic example for the continuum extrapolation of the two correlators at a given length $g_{3 \mathrm{~d}}^{2} L=1.0$. We clearly see that $\mathcal{O}(a)$ contributions are still present, but the consideration of parabolae with joint linear coefficients according to (3.2) and (3.3) results in curves that fit our data reasonably well. The obtained continuum values and their respective quadratic coefficients are close to each other, but can still be distinguished.

We considered four $(x, y)$ pairs, each at eight separations $L$ for the $\operatorname{Tr}\left\langle E(L) U E(0) U^{-1}\right\rangle$ correlator and seven separations for the $\operatorname{Tr}\left\langle B(L) U B(0) U^{-1}\right\rangle$ correlator, and at seven different lattice spacings. (Note that not every lattice spacing is useful for each length $L$; and we cannot evaluate the $\operatorname{Tr}\left\langle B(L) U B(0) U^{-1}\right\rangle$ correlator at the smallest $L$ value because as noted before, the clover $B$ operator is rather large, requiring larger $L / a$ values before one enters the continuum regime.) For each $(x, y)$ pair and separation, the $\operatorname{Tr}\left\langle E(L) U E(0) U^{-1}\right\rangle$ and $\operatorname{Tr}\left\langle B(L) U B(0) U^{-1}\right\rangle$ correlators are each continuum extrapolated, fitting to a constant and quadratic term, plus linear terms determined by the three global parameters $Z_{\mathrm{E}}, Z B$, and $Z_{\mathrm{P}}$. The likelihood of the grand continuum extrapolation was reasonable with a $\chi^{2} \approx 270$ on $276-123=153$ degrees of freedom.

\section{Integration of lattice data}

Even without the contributions from the $4 \mathrm{D}$ to $3 \mathrm{D}$ matching calculation, we can still attempt to perform the integral shown in Eq. (2.6), at least for $L$ above some small- $L$ cutoff. Our lattice data are available only within a finite range of separations $g_{3 \mathrm{~d}}^{2} L$. We integrate these data using the trapezoidal rule. Ideally, we would want data at a densely spaced set of distances $L$, but this is impractical because the $L$ spacing is limited by the lattice spacings of the coarsest lattices used. Also, using denser-spaced $L$ values would not actually help very much, because the different $L$-value data are from the same simulations, and data at nearby separations $L$ are highly correlated. Nevertheless, the finite spacing in $g_{3 \mathrm{~d}}^{2} L$ introduces another error in $Z_{\mathrm{g}}$ which we have to bear in mind. We choose to measure $\operatorname{Tr}\left\langle E(L) U E(0) U^{-1}\right\rangle$ at $g_{3 \mathrm{~d}}^{2} L=0.25,0.5,0.75,1.0,1.5,2.0,2.5,3.0$ and $\operatorname{Tr}\left\langle B(L) U B(0) U^{-1}\right\rangle$ at $g_{3 \mathrm{~d}}^{2} L=0.5,0.75,1.0,1.5,2.0,2.5$, 3.0, where we elaborate on this choice in the Appendix. We will eventually find that using the trapezoid rule only introduces a subdominant discrete-integration error.

\section{Integration of the tails}

The correlator (1.5) requires an integration of the condensate up to infinite separations. Since the signal-tonoise ratio drops dramatically with increasing $L$, correlators 
at separations beyond $g_{3 \mathrm{~d}}^{2} L \geq 3.0$ are not determined robustly. Nevertheless, knowing the analytical form of the correlators at large separations $L$, we can fit the tail to the largest data we have available. Similar things have been done, e.g., in the context of the hadronic contribution to the muon $g-2$-factor [52]. However, their considerations cannot be precisely applied in our case since we do not know the exact form of the higher excited states. For the magnetic contribution, Laine and Philipsen [53] conjecture a functional form

$\operatorname{Tr}\left\langle F^{x z}(L) U_{\mathrm{F}}(L, 0) F_{x z} U_{\mathrm{F}}^{-1}(L, 0)\right\rangle=\frac{A}{g_{3 \mathrm{~d}}^{4} L^{2}} \mathrm{e}^{-B g_{3 \mathrm{~d}}^{2} L}$.

Even though they consider a conventional Wilson line connecting the two $B$-fields, this form is still applicable. However, in our case, the coefficient $B$ has a valid continuum limit, rather than having logarithmically UV divergent perimeter contributions. Each coefficient is nonperturbative; we cannot predict them.

Based on similar physical reasoning, and consistent with the free solution (2.7), we expect the functional form of the large-separation limit of $\operatorname{Tr}\left\langle E(L) U E(0) U^{-1}\right\rangle$ also to be (3.4), with different coefficients to be determined from the fit. We choose our fitting range for the large- $g_{3 \mathrm{~d}}^{2} L$-tail to be $[1.5,3.0]$. This is hard to justify a priori, since the correlation length above which the lower bound of the interval must be precisely $\xi \sim 1 / m_{\infty}$. However, we will eventually find that $1.5 \gg g_{3 \mathrm{~d}}^{2} / m_{\infty}$.

At the other end, we do not have data at separations below certain minimal separations $g_{3 \mathrm{~d}}^{2} L_{\text {min }}^{\mathrm{E}}$ and $g_{3 \mathrm{~d}}^{2} L_{\min }^{\mathrm{B}}$. Below these minimal separations, one would have to connect to an EQCD perturbative expression. Since the (yet unknown) difference between the EQCD effective description and the "true" full-QCD version of (1.5) is expected to contribute significantly there, we leave the consideration of this region entirely for future study.

\section{RESULTS}

Before we give our numerical results, we present a thorough discussion of the multiple possible sources of both statistical and systematic errors throughout our calculation. As numerical results, we provide continuumextrapolated data for $\operatorname{Tr}\left\langle E(L) U E(0) U^{-1}\right\rangle$ and $\operatorname{Tr}\left\langle B(L) U B(0) U^{-1}\right\rangle$ in Figs. 3 and 4, the corresponding values can be found in Table II, and details about the simulations appear in Table III of the Appendix.

\section{A. Error estimation}

The estimation of the error has to take several sources into account. They are as follows:

(1) The statistical fluctuations of our data induced by the Monte Carlo simulation of the path integral.

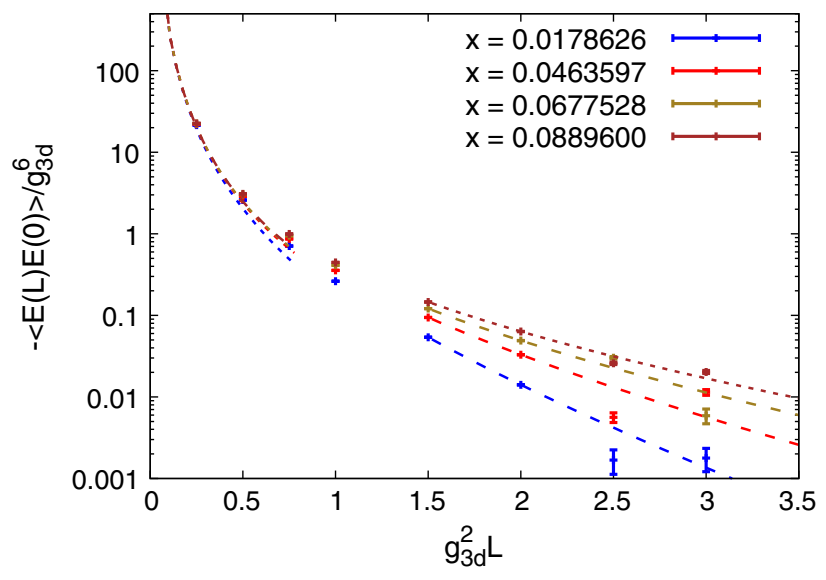

FIG. 3. Continuum-extrapolated version of $-\frac{\operatorname{Tr}\left\langle E(L) U E(0) U^{-1}\right\rangle}{g_{3 \mathrm{~d}}^{6}}$, connecting to the free solution (2.7) at small $g_{3 \mathrm{~d}}^{2} L$ and to an exponential tail of the form (3.4) with the coefficients determined from our data at $g_{3 \mathrm{~d}}^{2} L \leq 1.5$. The short-dashed lines indicate the respective free solutions corresponding to a pair of $x$ and $y$. The long-dashed lines refer to the respective fitted tail function of the form (3.4).

(2) The uncertainty in choosing the model for the long$L$-tail and in the determination of its parameters.

(3) The discretized evaluation of the $L \mathrm{~d} L$-integration.

(4) The matching to the perturbative solutions at small $g_{3 \mathrm{~d}}^{2} L$.

(5) The reliability of reducing full QCD to EQCD.

(6) The perturbative determination of $Z_{\mathrm{f}}$.

We will now discuss these aspects in detail, and give and explain quantitative estimates where possible; see Table II.

The Monte Carlo error of $\operatorname{Tr}\left\langle E(L) U E(0) U^{-1}\right\rangle$ and $\operatorname{Tr}\left\langle B(L) U B(0) U^{-1}\right\rangle$ can be determined by a standard

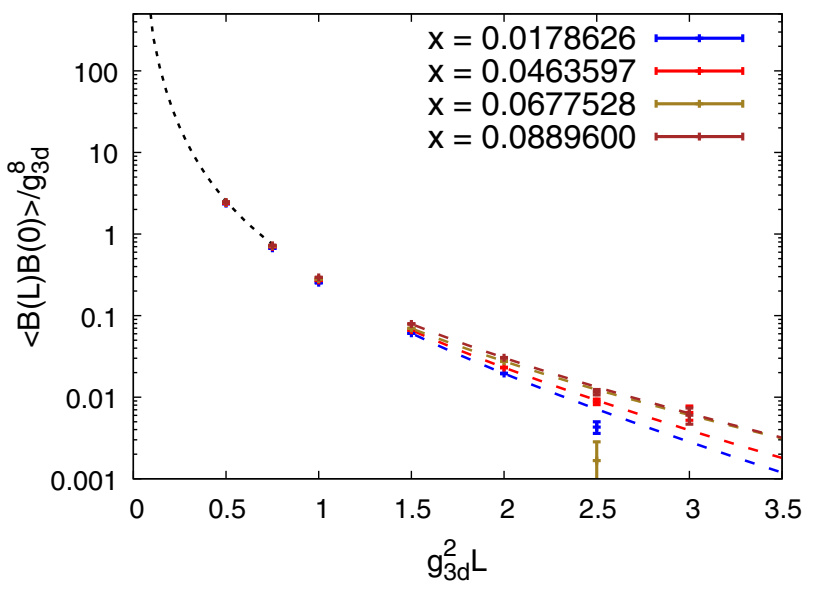

FIG. 4. Continuum-extrapolated version of $\frac{\operatorname{Tr}\left\langle B(L) U B(0) U^{-1}\right\rangle}{g_{3 \mathrm{~d}}^{8}}$, connecting to the free solution (2.8) at small $g_{3 \mathrm{~d}}^{2} L$ and to an exponential tail of the form (3.4) with the coefficients determined from our data at $g_{3 \mathrm{~d}}^{2} L \leq 1.5$. The black dashed line indicates the free solution that is independent of $x$ and $y$. The dashed lines in color refer to the respective fitted tail function of the form (3.4). 
TABLE II. Results for the correlators $\operatorname{Tr}\left\langle E(L) U E(0) U^{-1}\right\rangle / g_{3 \mathrm{~d}}^{6}$ and $\operatorname{Tr}\left\langle B(L) U B(0) U^{-1}\right\rangle / g_{3 \mathrm{~d}}^{8}$ at four temperatures over a range of separations. The sum of the integrated correlators according to (2.6) yields our estimate of $Z_{\mathrm{g}}$. We would like to point out once more that the integration still lacks UV contributions below $g_{3 \mathrm{~d}}^{2} L_{\min }^{\mathrm{E}}$ and $g_{3 \mathrm{~d}}^{2} L_{\mathrm{min}}^{\mathrm{B}}$, respectively.

\begin{tabular}{|c|c|c|c|c|}
\hline \multirow[b]{2}{*}{$g_{3 \mathrm{~d}}^{2} b_{\perp}$} & \multicolumn{2}{|c|}{$x=0.08896 y=0.452423 N_{f}=3,250 \mathrm{MeV}$} & \multicolumn{2}{|c|}{$x=0.0677528 y=0.586204 N_{f}=3,500 \mathrm{MeV}$} \\
\hline & $\langle E E\rangle$ & $\langle B B\rangle$ & $\langle E E\rangle$ & $\langle B B\rangle$ \\
\hline 0.25 & $-22.6327(61)$ & $\ldots$ & $-22.5353(55)$ & \\
\hline 0.50 & $-3.09410(94)$ & $2.47307(55)$ & $-3.03182(92)$ & $2.44142(45)$ \\
\hline 0.75 & $-0.99544(65)$ & $0.72585(86)$ & $-0.95011(36)$ & $0.71669(64)$ \\
\hline 1.0 & $-0.44656(16)$ & $0.29402(11)$ & $-0.41227(15)$ & $0.28046(11)$ \\
\hline 1.5 & $-0.14590(13)$ & $0.07913(13)$ & $-0.12111(18)$ & $0.06973(21)$ \\
\hline 2.0 & $-0.06351(19)$ & $0.03045(21)$ & $-0.04909(17)$ & $0.02757(22)$ \\
\hline 2.5 & $-0.02583(87)$ & $0.01153(88)$ & $-0.03021(99)$ & $0.0017(12)$ \\
\hline 3.0 & $-0.02027(89)$ & $0.0060(13)$ & $-0.0059(12)$ & $-0.0045(15)$ \\
\hline \multirow[t]{2}{*}{$\begin{array}{l}\frac{Z_{g}}{g_{3 \mathrm{~d}}^{4}} \\
\end{array}$} & \multicolumn{2}{|c|}{$0.731(1)(13)(2)$} & \multicolumn{2}{|c|}{$0.714(1)(14)(2)$} \\
\hline & \multicolumn{2}{|c|}{$x=0.0463597 y=0.823449 N_{f}=4,1 \mathrm{GeV}$} & \multicolumn{2}{|c|}{$x=0.0178626 y=1.64668 N_{f}=5,100 \mathrm{GeV}$} \\
\hline$g_{3 \mathrm{~d}}^{2} b_{\perp}$ & $\langle E E\rangle$ & $\langle B B\rangle$ & $\langle E E\rangle$ & $\langle B B\rangle$ \\
\hline 0.25 & $-22.1815(55)$ & & $-21.5097(53)$ & \\
\hline 0.50 & $-2.87898(91)$ & $2.40519(46)$ & $-2.59674(81)$ & $2.35855(48)$ \\
\hline 0.75 & $-0.86771(60)$ & $0.69914(72)$ & $-0.70851(37)$ & $0.66863(53)$ \\
\hline 1.0 & $-0.35581(13)$ & $0.26957(11)$ & $-0.26206(10)$ & $0.25329(10)$ \\
\hline 1.5 & $-0.09430(13)$ & $0.06631(16)$ & $-0.05388(10)$ & $0.06072(15)$ \\
\hline 2.0 & $-0.03296(15)$ & $0.02311(18)$ & $-0.01410(10)$ & $0.01952(15)$ \\
\hline 2.5 & $-0.00562(18)$ & $0.00879(67)$ & $-0.00168(56)$ & $0.00430(69)$ \\
\hline 3.0 & $-0.01139(90)$ & $0.0065(13)$ & $-0.00178(56)$ & $-0.00151(75)$ \\
\hline$\frac{Z_{g}}{g_{3 \mathrm{~d}}^{4}}$ & \multicolumn{2}{|c|}{$0.6502(8)(71)(8)$} & \multicolumn{2}{|c|}{$0.5711(7)(45)(1)$} \\
\hline
\end{tabular}

binning and Jackknife analysis. Since the continuum extrapolation was performed in a grand fit, one has to account for correlations of all lattices at all separations $g_{3 \mathrm{~d}}^{2} L$. Even if their $g_{3 \mathrm{~d}}^{2} a$ naively would not contribute, it does so via its influence on the values of $Z_{\mathrm{P}}, Z_{\mathrm{B}}$, and $Z_{\mathrm{E}}$. This error, $\Delta_{M C}$, is given in the first brackets in Table II.

Another dominantly statistical error is created when we match the tails of our models for $\operatorname{Tr}\left\langle E(L) U E(0) U^{-1}\right\rangle$ and $\operatorname{Tr}\left\langle B(L) U B(0) U^{-1}\right\rangle$; see (3.4). To be precise, the origin of this error is twofold; the assumption of the model itself introduces a systematic error, and the determination of the model parameters from our data at large $g_{3 \mathrm{~d}}^{2} L$ generates a statistical error. A valid estimate for this is provided by determining the coefficients in (3.4) by a fit, performing the integral, repeating this procedure for each Jackknife bin, and running the standard Jackknife analysis over the different values for the tail integral. This procedure includes the correlations between the fitting parameters in Eq. (3.4). In practice, we found that the statistical error amounts to about $100 \%$ of the correction induced by the large $-g_{3 \mathrm{~d}}^{2} L$ tail, which seems to be a reasonable estimate of the overall error of the large- $g_{3 \mathrm{~d}}^{2} L$-integration. This error, $\Delta_{\mathrm{LT}}$, is given in the second brackets of Table II.

Our data in the available window were integrated using the trapezoidal rule. The remainder $\Delta_{\mathrm{TR}}$ of such a discretized integration bounded by $g_{3 \mathrm{~d}}^{2} L_{\min }$ and $g_{3 \mathrm{~d}}^{2} L_{\text {max }}$ reads

$$
\Delta_{\mathrm{TR}}=\frac{g_{3 \mathrm{~d}}^{2} L_{\max }-g_{3 \mathrm{~d}}^{2} L_{\min }}{12} \max _{g_{3 \mathrm{~d}}^{2} L}\left|I^{\prime \prime}\left(g_{3 \mathrm{~d}}^{2} L\right)\right|,
$$

where the maximum of the second derivative of the integrand $I$ in (2.3) within the interval $\left[g_{3 \mathrm{~d}}^{2} L_{\min }, g_{3 \mathrm{~d}}^{2} L_{\max }\right]$ has to be found, involving the additional factor of $g_{3 \mathrm{~d}}^{2} L$ from the $L \mathrm{~d} L$-integration. This is tricky since the overall functional form of our data is precisely what we do not know. However, we know the functional form of the tails which we take as a basis. This might not be a rigorous choice, but we will see that this error is in any case subdominant compared to other error sources. For this consideration, we extend the range of validity of the free solutions up to $g_{3 \mathrm{~d}}^{2} L=1.0$ and the range of validity of the large- $g_{3 \mathrm{~d}}^{2} L$-tails down to the same value. Consequently, we have three trapezoids of length $\delta\left(g_{3 \mathrm{~d}}^{2} L\right)=0.25$ with freesolution estimates of the second derivative and four trapezoids of length $\delta\left(g_{3 \mathrm{~d}}^{2} L\right)=0.5$ with tail estimates. We give this systematic error in the third brackets in Table II.

Furthermore, the dimensional reduction to EQCD also introduces errors. These errors are of two types. First, there 
is the precision with which the matching is computed for the Lagrangian of EQCD. These corrections are formally suppressed by $\mathcal{O}\left(g^{4}\right)$ relative to the leading behavior. Second, there is the accuracy of the matching for the specific operator we are considering. So far, this has only been performed at tree level, which as we have already noted is insufficient. A calculation at the next order would represent parametrically $\mathcal{O}\left(g^{4}\right)$ contributions to $Z_{\mathrm{g}}$, which is formally $\mathcal{O}\left(g^{2}\right)$ relative to the leading behavior. Without this matching, there are formally $\mathcal{O}\left(g^{2}\right)$ relative errors from the matching calculation; with an NLO calculation, this would be reduced to formally $\mathcal{O}\left(g^{4}\right)$ errors. The reason an NLO matching is needed is that nonperturbative EQCD effects are also formally of relative order $\mathcal{O}\left(g^{2}\right)$; therefore, without an NLO matching calculation for the operator, we have not improved the formal order of the uncertainty. It is difficult to estimate the size of these (systematic) matching errors. An NLO calculation would shed considerable light on this point.

Last but not least, the current perturbative determination of $Z_{\mathrm{f}}$ neglects terms suppressed by a factor of $g^{2}$. These could be determined in an NLO calculation of this condensate within the full 4D theory.

All in all, the values of $Z_{\mathrm{g}}$ in Table II are of the form

$$
\operatorname{Best} \text { estimate }\left(\Delta_{\mathrm{MC}}\right)\left(\Delta_{\mathrm{LT}}\right)\left(\Delta_{\mathrm{TR}}\right) \text {. }
$$

Here $\Delta_{\mathrm{MC}}, \Delta_{\mathrm{LT}}$, and $\Delta_{\mathrm{TR}}$ are, respectively, the errors from Monte Carlo statistics including the continuum extrapolation, the long-distance tail, and the use of the trapezoid rule.

The overall quantified error of our results is dominated by integration of the long-distance tail. This introduces a relative error of $\sim 1 \%$. However, we assume that analytical uncertainties like the perturbative matching of the action and the operator and the perturbative determination of $Z_{\mathrm{f}}$ would not allow for higher precision anyway, so an improvement is not mandatory.

\section{B. Numerical results}

The plots Figs. 3 and 4 show agreement of our continuum data with the free solutions from (2.7) and (2.8) at small $g_{3 \mathrm{~d}}^{2} L$. Take note that, in contrast to $\operatorname{Tr}\left\langle E(L) U E(0) U^{-1}\right\rangle$, we do not have data for $\operatorname{Tr}\left\langle B(L) U B(0) U^{-1}\right\rangle$ at $g_{3 \mathrm{~d}}^{2} L=0.25$ since the discretization effects are more severe for this operator and we did not compute fine enough lattices to perform a valid continuum extrapolation at this distance. At the other end of the range, we fit the functional form (3.4) to the correlators through the last four points. The fit is dominated by the two innermost of these four points since the signal-to-noise-ratio drops dramatically at increasing $g_{3 \mathrm{~d}}^{2} L$. In turn, this phenomenon leads to some of the points in Fig. 4 at $g_{3 \mathrm{~d}}^{2} L=3.0$ even being negative and therefore not being displayed in the logarithmically scaled plot. As we can see from their values in Table II, they are still consistent with 0 within a few standard deviations.

We see in both plots that the dependence on the EQCD parameters ( $x$ and $y$ from Table $\mathrm{I}$ ) diminishes in going to small $g_{3 \mathrm{~d}}^{2} L$. For $\operatorname{Tr}\left\langle B(L) U B(0) U^{-1}\right\rangle$ in Fig. 4, this becomes evident by looking at the free solution (2.8) which has neither a dependence on $x$ nor on $y$ and is jointly approximated by all four scenarios. For Fig. 3 displaying $\operatorname{Tr}\left\langle E(L) U E(0) U^{-1}\right\rangle$, the situation is less obvious. Expanding the exponential in (2.7) for small $m_{\mathrm{D}} L$, we see that the dependence on $m_{\mathrm{D}}$, or $\sqrt{y}$, respectively, comes with a suppression of $\mathcal{O}\left(L^{2}\right)$ and therefore becomes negligible at small distances.

In both cases, we observe a strict hierarchy: the correlators $-\operatorname{Tr}\left\langle E(L) U E(0) U^{-1}\right\rangle$ and $\operatorname{Tr}\left\langle B(L) U B(0) U^{-1}\right\rangle$ grow with increasing $x$ (corresponding to decreasing the temperature). We also find that $-\operatorname{Tr}\left\langle E(L) U E(0) U^{-1}\right\rangle$ and $\operatorname{Tr}\left\langle B(L) U B(0) U^{-1}\right\rangle$ become closer to each other as one increases the temperature, which is expected due to the screening mass $m_{\mathrm{D}}$ increasing with temperature and reducing scalar correlations over large distances.

Adding $\operatorname{Tr}\left\langle E(L) U E(0) U^{-1}\right\rangle$ and $\operatorname{Tr}\left\langle B(L) U B(0) U^{-1}\right\rangle$ and integrating according to (2.6), as discussed in the previous section, one observes that $\operatorname{Tr}\left\langle E(L) U E(0) U^{-1}\right\rangle$ outweighs $\operatorname{Tr}\left\langle B(L) U B(0) U^{-1}\right\rangle$ and therefore drives the integral negative in all four scenarios. Multiplying by $-\frac{2 \cdot 2}{d_{\mathrm{A}}}=-\frac{1}{2}$, one indeed obtains a positive $Z_{\mathrm{g}}$.

Our results for $Z_{\mathrm{g}} / g_{3 \mathrm{~d}}^{4}$, shown in Table II, are significantly larger than the leading-order contribution from Eq. (1.9). However, note that we perform the $\operatorname{Tr}\left\langle E(L) U E(0) U^{-1}\right\rangle$ integration, which contributes positively, down to a smaller cutoff than the $\operatorname{Tr}\left\langle B(L) U B(0) U^{-1}\right\rangle$ integration, which contributes negatively. This mismatch dominates the presented result. However, we still present the result in this way, because it incorporates all results for which our numerical treatment gives statistically useful information. This mismatch can be fixed together with the incorporation of the NLO matching, which we expect to give a procedure for incorporating the missing short-distance information.

\section{CONCLUSION AND OUTLOOK}

In the present work, we have shown how to compute the asymptotic masses $m_{\infty}^{2}$ in QCD at high temperature nonperturbatively. We showed that the real-time continuum expression, Eqs. (1.4) and (1.5), can be matched to EQCD operators, Eq. (2.3), to compute the crucial nonperturbative infrared contributions. We then evaluated these operators numerically within EQCD on a 3D lattice, performing a precise extrapolation to the continuum limit. Our data are presented in Table II, and we discussed a procedure for numerically integrating these data to determine the IR contribution to the asymptotic masses, and for assessing all sources of statistical and systematic error in using the EQCD data. 
Unfortunately, we find that to use the numerical results properly, we need to complete the matching calculation for the operators of Eq. (1.5) between full QCD and EQCD a next-to-leading order in the perturbative expansion. Therefore, we are in the unusual situation that the numerical study of EQCD is currently ahead of the analytical understanding of the matching calculation. Such a matching calculation would improve the short-distance part of the EQCD calculation by providing an NLO result for the small-separation correlation functions, and it would ensure that all effects up to the desired $m_{\infty}^{2} \sim g^{4} T^{2}$ level of accuracy are accounted for. In terms of future improvements, this is clearly the most pressing, since without this matching calculation our results cannot yet be used. Further improvements to our numerical calculation are possible, in particular by sharpening the error bars and computing down to shorter distances, but generally the data appear to be in good shape and the matching calculation is the most urgent need.

Together with the transverse collision kernel $C\left(\boldsymbol{b}_{\perp}\right)$ from [43], the then-complete- $m_{\infty}^{2}$ will hopefully contribute significantly to understanding how a jet is modified by the medium that it traverses. We are eager to see the impact of both results on phenomenological transport calculations and how they match experimental observations.

\section{ACKNOWLEDGMENTS}

This work was supported by the Deutsche Forschungsgemeinschaft (DFG, German Research Foundation)Project No. 315477589-CRC TRR 211. Calculations for this research were conducted on the Lichtenberg high performance computer of the TU Darmstadt. N. S. acknowledges support from Academy of Finland Grants No. 267286 and No. 320123. We thank Sören Schlichting and Ismail Soudi for fruitful discussions on related topics in the course of which the idea for this project was born.

\section{APPENDIX: SIMULATION PARAMETERS}

The parameters of our simulations can be found in Table III. The volumes were chosen large enough such that the suppression of finite-volume effects according to [54] was maintained. All separations of both $\operatorname{Tr}\left\langle E(L) U E(0) U^{-1}\right\rangle$

TABLE III. Parameters for all EQCD multilevel simulations.

\begin{tabular}{|c|c|c|c|c|c|}
\hline$g_{3 \mathrm{~d}}^{2} a$ & $x_{\text {cont }}$ & $y_{\text {cont }}$ & $N_{\mathrm{x}} N_{\mathrm{y}} N_{\mathrm{z}}$ & $L / a$ & Statistics \\
\hline $1 / 4$ & 0.08896 & 0.452423 & $24^{2} \times 48$ & $4,6,8,10,12$ & 94560 \\
\hline $1 / 6$ & 0.08896 & 0.452423 & $36^{2} \times 72$ & $6,12,18$ & 88740 \\
\hline $1 / 8$ & 0.08896 & 0.452423 & $48^{2} \times 96$ & $4,6,8,12,16,20,24$ & 61420 \\
\hline $1 / 12$ & 0.08896 & 0.452423 & $72^{2} \times 144$ & $6,12,18,24,30,36$ & 8820 \\
\hline $1 / 16$ & 0.08896 & 0.452423 & $96^{2} \times 192$ & $4,8,12,16,24,32,40$ & 2400 \\
\hline $1 / 24$ & 0.08896 & 0.452423 & $144^{2} \times 288$ & $6,12,18,24$ & 480 \\
\hline $1 / 32$ & 0.08896 & 0.452423 & $192^{2} \times 384$ & $4,8,16,24,32$ & 200 \\
\hline $1 / 4$ & 0.0677528 & 0.586204 & $24^{2} \times 48$ & $4,6,8,10,12$ & 50200 \\
\hline $1 / 6$ & 0.0677528 & 0.586204 & $36^{2} \times 72$ & $6,12,18$ & 29480 \\
\hline $1 / 8$ & 0.0677528 & 0.586204 & $48^{2} \times 96$ & $4,6,8,12,16,20,24$ & 23940 \\
\hline $1 / 12$ & 0.0677528 & 0.586204 & $72^{2} \times 144$ & $6,12,18,24,30,36$ & 8680 \\
\hline $1 / 16$ & 0.0677528 & 0.586204 & $96^{2} \times 192$ & $4,8,12,16,24,32,40$ & 3600 \\
\hline $1 / 24$ & 0.0677528 & 0.586204 & $144^{2} \times 288$ & $6,12,18,24$ & 480 \\
\hline $1 / 32$ & 0.0677528 & 0.586204 & $192^{2} \times 384$ & $4,8,16,24,32$ & 360 \\
\hline $1 / 4$ & 0.0463597 & 0.823449 & $24^{2} \times 48$ & $4,6,8,10,12$ & 62350 \\
\hline $1 / 6$ & 0.0463597 & 0.823449 & $36^{2} \times 72$ & $6,12,18$ & 36440 \\
\hline $1 / 8$ & 0.0463597 & 0.823449 & $48^{2} \times 96$ & $4,6,8,12,16,20,24$ & 29380 \\
\hline $1 / 12$ & 0.0463597 & 0.823449 & $72^{2} \times 144$ & $6,12,18,24,30,36$ & 8780 \\
\hline $1 / 16$ & 0.0463597 & 0.823449 & $96^{2} \times 192$ & $4,8,12,16,24,32,40$ & 3760 \\
\hline $1 / 24$ & 0.0463597 & 0.823449 & $144^{2} \times 288$ & $6,12,18,24$ & 460 \\
\hline $1 / 32$ & 0.0463597 & 0.823449 & $192^{2} \times 384$ & $4,8,16,24,32$ & 300 \\
\hline $1 / 4$ & 0.0178626 & 1.64668 & $24^{2} \times 48$ & $4,6,8,10,12$ & 62340 \\
\hline $1 / 6$ & 0.0178626 & 1.64668 & $36^{2} \times 72$ & $6,12,18$ & 36500 \\
\hline $1 / 8$ & 0.0178626 & 1.64668 & $48^{2} \times 96$ & $4,6,8,12,16,20,24$ & 29580 \\
\hline $1 / 12$ & 0.0178626 & 1.64668 & $72^{2} \times 144$ & $6,12,18,24,30,36$ & 8740 \\
\hline $1 / 16$ & 0.0178626 & 1.64668 & $96^{2} \times 192$ & $4,8,12,16,24,32,40$ & 3700 \\
\hline $1 / 24$ & 0.0178626 & 1.64668 & $144^{2} \times 288$ & $6,12,18,24$ & 480 \\
\hline $1 / 32$ & 0.0178626 & 1.64668 & $192^{2} \times 384$ & $4,8,16,24,32$ & 300 \\
\hline
\end{tabular}


and $\operatorname{Tr}\left\langle B(L) U B(0) U^{-1}\right\rangle$ were measured on the same lattice configurations, introducing correlations between the different $g_{3 \mathrm{~d}}^{2} L$ that had to be accounted for in the error analysis. The measurement of the very smallest separation, $g_{3 \mathrm{~d}}^{2} L=$ 0.125 on the $g_{3 \mathrm{~d}}^{2} a=1 / 32$-lattices could not contribute to any continuum extrapolation since data were available at that particular spacing only. We used these data just for cross-checking. The statistics for the $T=250 \mathrm{MeV}$ lattices was mostly higher since we used this case to investigate how large separations $g_{3 \mathrm{~d}}^{2} L$ could be taken into account. On each of the other temperatures, about 12000 CPU.hrs on Intel-Xeon E5-2670-processors were spent.
[1] I. Arsene et al., Quark gluon plasma and color glass condensate at RHIC? The perspective from the BRAHMS experiment, Nucl. Phys. A757, 1 (2005).

[2] B. B. Back et al., The PHOBOS perspective on discoveries at RHIC, Nucl. Phys. A757, 28 (2005).

[3] J. Adams et al., Experimental and theoretical challenges in the search for the quark gluon plasma: The STAR Collaboration's critical assessment of the evidence from RHIC collisions, Nucl. Phys. A757, 102 (2005).

[4] K. Adcox et al., Formation of dense partonic matter in relativistic nucleus-nucleus collisions at RHIC: Experimental evaluation by the PHENIX collaboration, Nucl. Phys. A757, 184 (2005).

[5] E. V. Shuryak, Theory of hadronic plasma, Zh. Eksp. Teor. Fiz. 74, 408 (1978) [Sov. Phys. JETP 47, 212 (1978)].

[6] J. I. Kapusta, Quantum chromodynamics at high temperature, Nucl. Phys. B148, 461 (1979).

[7] T. Toimela, The next term in the thermodynamic potential of QCD, Phys. Lett. 124B, 407 (1983).

[8] P. B. Arnold and C.-x. Zhai, The three loop free energy for high temperature QED and QCD with fermions, Phys. Rev. D 51, 1906 (1995).

[9] C.-x. Zhai and B. M. Kastening, The free energy of hot gauge theories with fermions through $g^{5}$, Phys. Rev. D 52, 7232 (1995).

[10] K. Kajantie, M. Laine, K. Rummukainen, and Y. Schroder, The pressure of hot QCD up to g6 $\ln (1 / \mathrm{g})$, Phys. Rev. D 67, 105008 (2003).

[11] A. Bazavov et al., Equation of state in $(2+1)$-flavor QCD, Phys. Rev. D 90, 094503 (2014).

[12] S. Borsanyi, Z. Fodor, C. Hoelbling, S. D. Katz, S. Krieg, and K. K. Szabo, Full result for the QCD equation of state with $2+1$ flavors, Phys. Lett. B 730, 99 (2014).

[13] A. Bazavov et al., Skewness, kurtosis, and the fifth and sixth order cumulants of net baryon-number distributions from lattice QCD confront high-statistics STAR data, Phys. Rev. D 101, 074502 (2020).

[14] H. B. Meyer, A calculation of the shear viscosity in SU(3) gluodynamics, Phys. Rev. D 76, 101701 (2007).

[15] J. Ghiglieri and H. J. Kim, Transverse momentum broadening and collinear radiation at NLO in the $\mathcal{N}=4 \mathrm{SYM}$ plasma, J. High Energy Phys. 12 (2018) 049.

[16] M. Cè, T. Harris, H. B. Meyer, A. Steinberg, and A. Toniato, The rate of photon production in the quark-gluon plasma from lattice QCD (2020).

[17] J. Ghiglieri, J. Hong, A. Kurkela, E. Lu, G. D. Moore, and D. Teaney, Next-to-leading order thermal photon production in a weakly coupled quark-gluon plasma, J. High Energy Phys. 05 (2013) 010.

[18] J. Ghiglieri, G. D. Moore, and D. Teaney, Jet-medium interactions at NLO in a weakly-coupled quark-gluon plasma, J. High Energy Phys. 03 (2016) 095.

[19] D. J. Gross and F. Wilczek, Ultraviolet Behavior of Non-Abelian Gauge Theories, Phys. Rev. Lett. 30, 1343 (1973).

[20] H. D. Politzer, Reliable Perturbative Results for Strong Interactions?, Phys. Rev. Lett. 30, 1346 (1973).

[21] B. G. Zakharov, Fully quantum treatment of the LandauPomeranchuk-Migdal effect in QED and QCD, JETP Lett. 63, 952 (1996).

[22] B. G. Zakharov, Light cone path integral approach to the Landau-Pomeranchuk-Migdal effect, Yad. Fiz. 61, 924 (1998) [Phys. At. Nucl. 61, 838 (1998)].

[23] B. G. Zakharov, Radiative energy loss of high-energy quarks in finite size nuclear matter and quark-gluon plasma, JETP Lett. 65, 615 (1997).

[24] R. Baier, D. Schiff, and B. G. Zakharov, Energy loss in perturbative QCD, Annu. Rev. Nucl. Part. Sci. 50, 37 (2000).

[25] V. V. Klimov, Spectrum of elementary fermi excitations in quark gluon plasma. (In Russian), Sov. J. Nucl. Phys. 33, 934 (1981).

[26] H. A. Weldon, Covariant calculations at finite temperature: The relativistic plasma, Phys. Rev. D 26, 1394 (1982).

[27] V. V. Klimov, Collective excitations in a hot quark gluon plasma, Sov. Phys. JETP 55, 199 (1982).

[28] H. A. Weldon, Effective fermion masses of order gT in high temperature gauge theories with exact chiral invariance, Phys. Rev. D 26, 2789 (1982).

[29] E. Braaten and R. D. Pisarski, Resummation and Gauge Invariance of the Gluon Damping Rate in Hot QCD, Phys. Rev. Lett. 64, 1338 (1990).

[30] E. Braaten and R. D. Pisarski, Calculation of the gluon damping rate in hot QCD, Phys. Rev. D 42, 2156 (1990).

[31] J.-P. Blaizot, A. Ipp, A. Rebhan, and U. Reinosa, Asymptotic thermal quark masses and the entropy of QCD in the large-N(f) limit, Phys. Rev. D 72, 125005 (2005).

[32] E. Braaten and R. D. Pisarski, Simple effective Lagrangian for hard thermal loops, Phys. Rev. D 45, 1827 (1992).

[33] S. Caron-Huot, On supersymmetry at finite temperature, Phys. Rev. D 79, 125002 (2009).

[34] A. D. Linde, Infrared problem in thermodynamics of the Yang-Mills gas, Phys. Lett. 96B, 289 (1980). 
[35] T. Appelquist and R. D. Pisarski, High-temperature YangMills theories and three-dimensional quantum chromodynamics, Phys. Rev. D 23, 2305 (1981).

[36] S. Nadkarni, Dimensional reduction in hot QCD, Phys. Rev. D 27, 917 (1983).

[37] E. Braaten and A. Nieto, Effective field theory approach to high temperature thermodynamics, Phys. Rev. D 51, 6990 (1995).

[38] S. Caron-Huot, $\mathrm{O}(\mathrm{g})$ plasma effects in jet quenching, Phys. Rev. D 79, 065039 (2009).

[39] M. Panero, K. Rummukainen, and A. Schäfer, Lattice Study of the Jet Quenching Parameter, Phys. Rev. Lett. 112, 162001 (2014).

[40] G. D. Moore and N. Schlusser, Transverse momentum broadening from the lattice, Phys. Rev. D 101, 014505 (2020); Erratum, Phys. Rev. D 101, 059903 (2020).

[41] M. Laine and Y. Schroder, Two-loop QCD gauge coupling at high temperatures, J. High Energy Phys. 03 (2005) 067.

[42] K. Kajantie, M. Laine, A. Rajantie, K. Rummukainen, and M. Tsypin, The phase diagram of three-dimensional SU(3) + adjoint Higgs theory, J. High Energy Phys. 11 (1998) 011.

[43] G. D. Moore and N. Schlusser, Full O(a) improvement in electrostatic QCD, Phys. Rev. D 100, 034510 (2019).

[44] M. Laine, What is the simplest effective approach to hot QCD thermodynamics?, in Proceedings, 5th Internationa Conference on Strong and Electroweak Matter (SEWM 2002): Heidelberg, Germany, 2002 (World Scientific, New York, 2003), pp. 137-146, https://doi.org/10.1142/5243.
[45] http://luscher.web.cern.ch/luscher/openQCD/index.html.

[46] G. D. Moore, O(a) errors in 3-D SU(N) Higgs theories, Nucl. Phys. B523, 569 (1998).

[47] M. Luscher and P. Weisz, Locality and exponential error reduction in numerical lattice gauge theory, J. High Energy Phys. 09 (2001) 010.

[48] H. B. Meyer, Locality and statistical error reduction on correlation functions, J. High Energy Phys. 01 (2003) 048.

[49] P. Weisz, Continuum limit improved lattice action for pure Yang-Mills theory. 1., Nucl. Phys. B212, 1 (1983).

[50] P. Weisz and R. Wohlert, Continuum limit improved lattice action for pure Yang-Mills theory. 2., Nucl. Phys. B236, 397 (1984); Erratum, Nucl. Phys. B247, 544 (1984).

[51] M. D’Onofrio, A. Kurkela, and G. D. Moore, Renormalization of null Wilson lines in EQCD, J. High Energy Phys. 03 (2014) 125.

[52] A. Gérardin, M. Cè, G. von Hippel, B. Hörz, H. B. Meyer, D. Mohler, K. Ottnad, J. Wilhelm, and H. Wittig, The leading hadronic contribution to $(g-2)_{\mu}$ from lattice QCD with $N_{\mathrm{f}}=2+1$ flavours of $\mathrm{O}(a)$ improved Wilson quarks, Phys. Rev. D 100, 014510 (2019).

[53] M. Laine and O. Philipsen, Gauge invariant scalar and field strength correlators in 3-D, Nucl. Phys. B523, 267 (1998).

[54] A. Hietanen, K. Kajantie, M. Laine, K. Rummukainen, and Y. Schroder, Three-dimensional physics and the pressure of hot QCD, Phys. Rev. D 79, 045018 (2009). 